\title{
El costo de la belleza: Comunicación de seis casos clínicos con infección por micobacterias atípicas secundario a inyecciones intradérmicas y reacción a un dermoimplante de origen desconocido
}

\author{
Alejandro Schcolnik-Cabrera', Elisa Vega-Memije², Rigoberto Hernández-Castro², \\ Roberto Arenas ${ }^{2}$ y Ramón Fernández-Martínez ${ }^{2}$
}

'PECEM, Facultad de Medicina, Universidad Nacional Autónoma de México.

${ }^{2}$ Hospital General "Dr. Manuel Gea González", Ciudad de México, México.

Conflictos de interés: Los autores declaran no contar con conflicto de interés alguno.

Financiamiento: Este trabajo fue realizado gracias a los fondos del Hospital General "Dr. Manuel Gea González" en la Ciudad de México, México.

Recibido: 19 de septiembre de 2018 Aceptado: 2 de septiembre de

Correspondencia a: Ramón Fernández-Martínez ramfer2@hotmail.com

\begin{abstract}
The cost of beauty: six cosmetological clinical cases due to atypical mycobacterial infection secondary to intradermal injections plus reaction to foreign dermal implant
\end{abstract}

We report six cases of female patients who, after the application by mesotherapy with platelet-rich plasma, as well as of an intradermal filler material of unknown origin, developed infection at the injection sites associated to Mycobacterium massiliense, as well as granuloma with reaction to foreign body. Although the cultures were negative, the identification of the microorganism was achieved by extraction of soft tissue DNA obtained by biopsy and sequencing the obtained product, with which the therapy was redirected against the particular species. Due to the great similarity in the culture between $M$. massiliense with the related species $M$. abscessus, to the required time for its growth, and to the different therapeutic response of each strain, molecular diagnostic techniques are a real option to consider to administer in an early way the appropriate treatment against the pathogen and prevent infection progression.

Keywords: Filler complications, mesotherapy; platelet-rich plasma; atypical mycobacterial infection.

Palabras clave: Complicaciones por rellenos inyectables; mesoterapia; plasma rico en plaquetas; infección por micobacterias atípicas.

\section{Introducción}

$\mathrm{C}$ ada vez son más frecuentes los procedimientos con fines estéticos que utilizan tratamientos con plasma autólogo rico en plaquetas (PRP). Estas sustancias se aplican con la técnica de mesoterapia, o en gel para relleno intradérmico o subcutáneo. La mesoterapia consiste en la aplicación de inyecciones intradérmicas con sustancias farmacológicas diluidas directamente al sitio que se va a tratar. Estos procedimientos no siempre se realizan siguiendo estándares de seguridad e higiene, e incluso son realizados por personal no médico. También es frecuente la aplicación de otras sustancias con las misma técnica, que no están aprobados por entes reguladores y sin registro sanitario ${ }^{1}$.

Asociado a estos procedimientos, se han descrito complicaciones como infecciones en piel y tejidos blandos. La enfermedad asociada a micobacterias no tuberculosas (MNTs) es el tipo de infección más común asociado a la mesoterapia, particularmente por Mycobacterium chelonae y Mycobacterium abscessus ${ }^{2}$. El diagnóstico microbiológico de estas infecciones es difícil, debido a la baja sensibilidad y especificidad de las técnicas empleadas para ello, como el cultivo en Löwenstein Jensen ${ }^{3}$, y el tiempo necesario para el crecimiento de este tipo de microorganismos ${ }^{4,5}$.

Las micobacteriosis atípicas suelen ser de larga evolución, y requieren terapia combinada de antimicrobianos, a dosis elevadas y por períodos prolongados. La sensibilidad de las MNT a los antimicrobianos difiere según la especie, por lo que lo ideal es identificar al microorganismo a este nivel, lo que puede conseguirse con técnicas moleculares, como la secuenciación de regiones génicas.

Comunicamos seis casos de mujeres que presentaron una reacción a cuerpo extraño por inyecciones intradérmicas con un material de relleno de naturaleza desconocida, y una infección de piel y tejidos blandos por MNT, secundario a PRP aplicado con la técnica de mesoterapia. El agente causal se logró identificar por biología molecular y secuenciación de los productos obtenidos. Dos casos fueron diagnosticados y tratados por los antecedentes y los cuadros clínicos. 


\section{Casos clínicos}

Fueron seis mujeres previamente sanas quienes, estudiando un curso de cosmetología, se realizaron inyecciones intradérmicas con un material de relleno de naturaleza desconocida, denominado "elastina", sin registro sanitario, así como con PRP con la técnica de mesoterapia. Entre 15 días y dos meses después de este procedimiento, presentaron lesiones en el área tratada, caracterizadas por eritema, pápulas, nódulos y placas de hasta $3 \mathrm{~cm}$ de diámetro, así como cicatrices, algunas de ellas con drenaje de material hemático y purulento, con dolor al tacto y compromiso del estado general (Figura 1). Las características clínicas, tratamiento y evolución de cada paciente se detallan en la Tabla 1 .

Todas las pacientes habían sido tratadas previamente sin éxito con antibacterianos, principalmente cefalosporinas, por lo que se sospechó una infección por micobacterias. Ninguna tenía diagnóstico de inmunodeficiencia ni co-morbilidades de importancia. Dos pacientes se rehusaron a cualquier tipo de procedimiento diagnóstico. De las cuatro restantes, se tomaron múltiples muestras de piel y tejidos blandos, las cuales fueron empleadas para realizar frotis con tinción de Ziehl-Neelsen y Fite-Faraco, para cultivos en agar de Löwenstein-Jensen, biopsia para estudio histopatológico con las mismas tinciones, y para extracción de ADN y reacción de polimerasa en cadena (RPC) con secuenciación de los productos obtenidos. Los resultados de los análisis se muestran en la Tabla 2.

El informe histopatológico mostró infiltrados inflamatorios, con células gigantes secundarias a reacción a cuerpo extraño, así como material basófilo, granulomas, y múltiples áreas con necrosis y vacuolas de diferente tamaño, correspondiente a material extraño con apariencia similar al aceite; algunas pacientes desarrollaron depósitos mucinosos. Las biopsias teñidas con Ziehl-Neelsen y Fite-Faraco no mostraron micobacterias ácido alcoholresistentes (Figura 2).

Se extrajo el ADN de los tejidos fijados en parafina con el kit comercial DNeasy (Qiagen, La Jolla, CA, USA). Se amplificó la subunidad ribosomal 16S con RPC, empleando los partidores (5'-gga-tcctttgatcctggctcaggac-3' y 5'-acttgacgtcgtccccaccttcctc-3') diseñados a partir de la secuencia ribosomal 16S de Nocardia asteroides, ATCC

\begin{tabular}{|c|c|c|c|c|c|c|}
\hline Paciente & $\begin{array}{l}\text { Edad } \\
\text { (años) }\end{array}$ & $\begin{array}{l}\text { Tiempo de } \\
\text { evolución }\end{array}$ & Signos y síntomas & Tratamiento & $\begin{array}{l}\text { Duración del } \\
\text { tratamiento (meses) }\end{array}$ & Secuelas \\
\hline 1 & 34 & 2 semanas & $\begin{array}{l}\text { Nódulos, pápulas, } \\
\text { fiebre }\end{array}$ & $\begin{array}{l}\text { Moxifloxacina, claritromicina, difenhi- } \\
\text { dramina, desbridamiento quirúrgico }\end{array}$ & 6 & Cicatrices \\
\hline 2 & 48 & 2 semanas & Nódulos & $\begin{array}{l}\text { Moxifloxacina, claritromicina, cotri- } \\
\text { moxazol }\end{array}$ & 5 & Cicatrices \\
\hline 3 & 28 & $24 \mathrm{~h}$ & Nódulos & Moxifloxacina, claritromicina & 5 & Cicatrices \\
\hline 4 & 46 & 8 semanas & Placas, fístulas & $\begin{array}{l}\text { Moxifloxacina, claritromicina, desbri- } \\
\text { damiento quirúrgico }\end{array}$ & 6 & Cicatrices \\
\hline 5 & 40 & 3 semanas & $\begin{array}{l}\text { Nódulos, fiebre, mal } \\
\text { estado general }\end{array}$ & $\begin{array}{l}\text { Moxifloxacina, claritromicina, cotri- } \\
\text { moxazol, kanamicina, prednisona, } \\
\text { desbridamiento quirúrgico }\end{array}$ & 6 & $\begin{array}{l}\text { Sensación quemante facial y } \\
\text { dolor en cicatrices, } \\
\text { compromiso del estado general }\end{array}$ \\
\hline 6 & 40 & 6 semanas & $\begin{array}{l}\text { Nódulos, eritema, } \\
\text { pápulas }\end{array}$ & $\begin{array}{l}\text { Moxifloxacina, claritromicina, cotri- } \\
\text { moxazol, azitromicina, montelukast }\end{array}$ & 7 & Angioedema \\
\hline
\end{tabular}

Tabla 2. Resultados de las pruebas de laboratorio

\begin{tabular}{|c|c|c|c|c|}
\hline Paciente (número) & Frotis & Cultivo & Biopsia & RPC \\
\hline 1 & No aceptó & No aceptó & No aceptó & No aceptó \\
\hline 2 & $(+)$ & $(-)$ & $(-)$ & $(-)$ \\
\hline 3 & No aceptó & No aceptó & No aceptó & No aceptó \\
\hline 4 & $(-)$ & $(-)$ & $(-)$ & $(+)$ \\
\hline 5 & $(-)$ & $(-)$ & $(-)$ & $(+)$ \\
\hline 6 & $(+)$ & $(-)$ & $(-)$ & $(-)$ \\
\hline
\end{tabular}




\section{Caso Clínico}

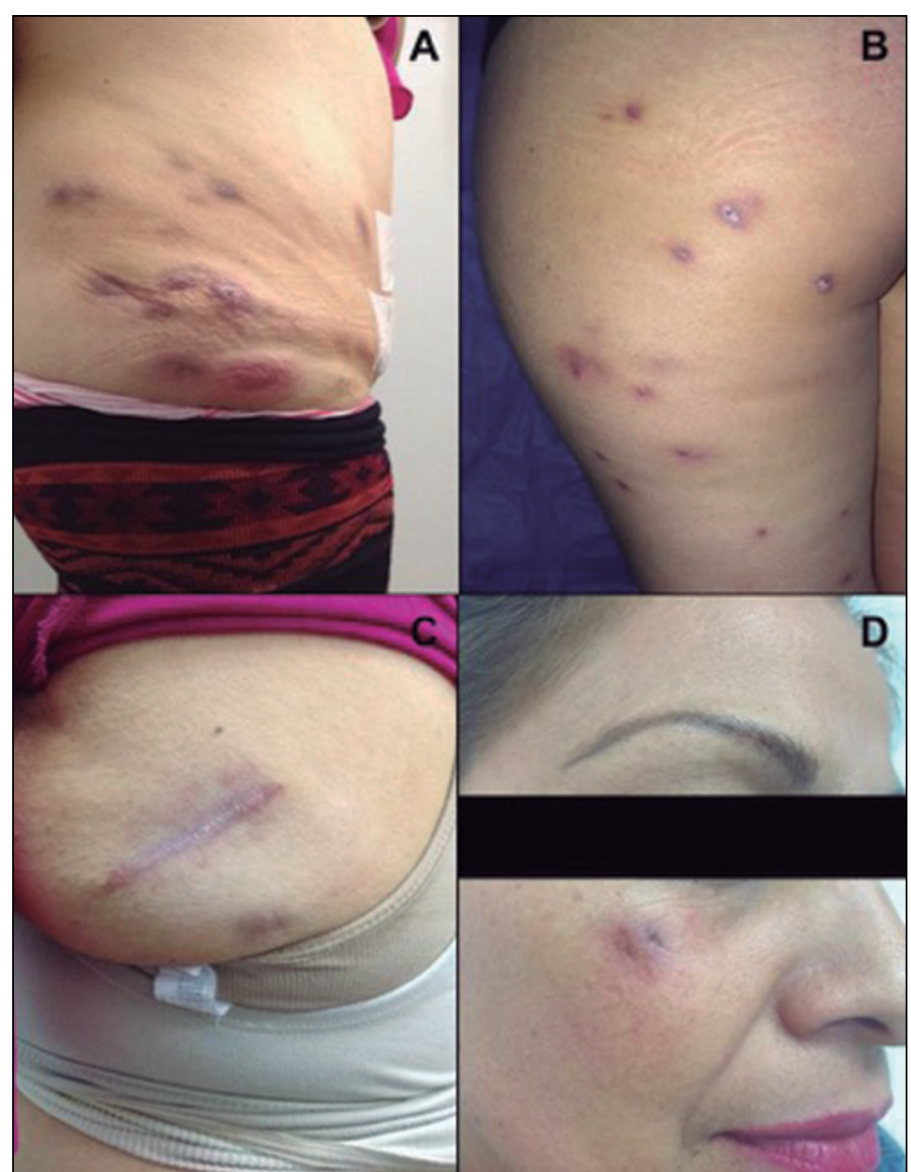

Figura 1. Hallazgos clínicos. A. Placas abdominales. B. Lesiones nodulares en glúteos y muslos. C y $\mathbf{D}$. Cicatrices y eritema secuelares.

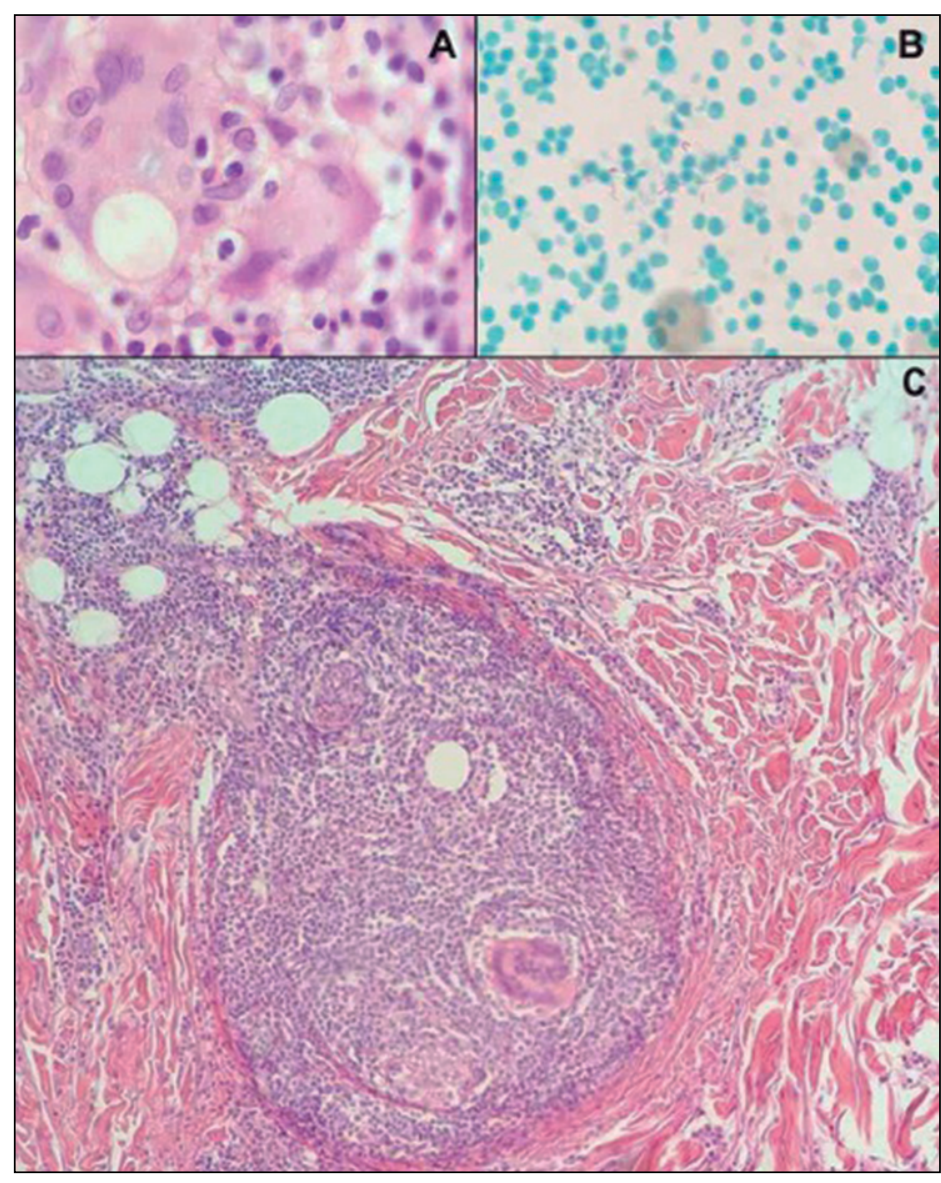

Figura 2. Hallazgos patológicos. A. Célula gigante incluyendo material extraño (40X, H\&E). B. Bacilos ácido-alcohol resistentes (100X, Ziehl-Neelsen). C. Lesión granulomatosa con material extraño (20X, H\&E).
49872 (número de acceso: AY191251), para amplificar un producto de 1.109 pares de bases. Las condiciones empleadas para la amplificación fueron: un ciclo de desnaturalización inicial a $95^{\circ} \mathrm{C}$ por $5 \mathrm{~min}$, seguido de 35 ciclos (desnaturalización, $30 \mathrm{seg}$ a $96^{\circ} \mathrm{C}$; alineación, 1 min a $70^{\circ} \mathrm{C}$; extensión, $1 \mathrm{~min}$ a $72^{\circ} \mathrm{C}$ ), con un ciclo de extensión final a $72^{\circ} \mathrm{C}$ por $5 \mathrm{~min}$. Los productos amplificados fueron visualizados en geles de agarosa al $1 \%$ teñidos con bromuro de etidio. Los productos de RPC se purificaron con el sistema comercial QIAquick PCR Purification Kit (Qiagen, La Jolla, CA, USA), siguiendo las instrucciones de los fabricantes.

La secuencia de los nucleótidos se determinó en ambas direcciones con la técnica Taq FS Dye Terminator Cycle Sequencing Fluorescence-Based Sequencing. La secuencia completa de los nucleótidos se analizó en el GenBank para buscar homologías en secuencia con Blast. La secuencia mostró 100\% de identidad con Mycobacterium massiliense.
Tras identificar al agente, los esquemas de tratamiento consistieron en una combinación de moxifloxacina 400 mg cada $12 \mathrm{~h}$, claritromicina $500 \mathrm{mg}$ cada $12 \mathrm{~h}$, y cotrimoxazol $800 \mathrm{mg} / 160 \mathrm{mg}$, cada $12 \mathrm{~h}$, durante 6 meses en promedio (Tabla 1). Una paciente requirió tratamiento por 6 semanas con kanamicina debido a ausencia de mejoría clínica, y en otra paciente se optó por emplear azitromicina por la misma causa. Un año después, una paciente persistió con angioedema y otra con deterioro del estado general y sensación urente en los sitios de lesión. El resto de pacientes, por motivos personales, decidieron no acudir a consultas posteriores de seguimiento.

\section{Discusión}

Los materiales de relleno no son inocuos y se han relacionado con diferentes reacciones adversas, generalmente inespecíficas. Dependiendo del momento en que 
ocurran, las complicaciones se dividen en inmediatas, tempranas y tardías, según suceden durante los primeros dos días, entre los 3 y 14 días, o tras dos semanas postinyección; respectivamente ${ }^{6}$. Las complicaciones tardías incluyen inflamación crónica, granulomas, infecciones por Mycobacterium spp., necrosis, osteomielitis crónica y muerte ${ }^{7}$. A diferencia de las infecciones asociadas al empleo de prótesis, la remoción completa de los materiales inyectables puede no ser posible y requieren de terapias más prolongadas 8 .

El PRP, reconocido como un volumen sanguíneo con concentración plaquetaria superior a la basal obtenido del mismo paciente a partir de una vena periférica, se encuentra enriquecido en factores de crecimiento y fibrina, y ha sido utilizado en la reparación de heridas ${ }^{9}$. Una técnica usada para la aplicación de PRP es la mesoterapia. Se han reportado complicaciones en su empleo, como reacciones alérgicas, dolor persistente, complicaciones neuromusculares en el sitio de inyección, ulceración, paniculitis con necrosis grasa e infecciones bacterianas ${ }^{10,11}$.

Aunque la tinción para bacilos ácido alcohol resistentes visualizados con microscopía de luz del material obtenido de los abscesos es la técnica más económica y rápida para determinar las infecciones por micobacterias, la RPC es la técnica que más ha evolucionado para el diagnóstico de infecciones por MNTs. De hecho, puede ser realizada directamente de los tejidos y sin necesidad de cultivos, para conocer, junto con secuenciación del ADN, el género y la especie de los microorganismos implicados ${ }^{12,13}$. Además, aunque las MNTs pueden crecer en diferentes medios de cultivo, como agar sangre, Sabouraud, Löwenstein-Jensen y Ogawa ${ }^{4}$, no siempre es posible su crecimiento. Como se ha reportado previamente, el medio LöwensteinJensen presenta una sensibilidad de $63,9-79,5 \%$ para la detección de micobacterias, y su tasa de contaminación es del $27,2 \%{ }^{3}$. En cuanto a la tinción de Ziehl-Neelsen, se ha demostrado una sensibilidad de $40 \%$ para identificar micobacterias, en comparación con la RPC, que asciende a $83 \%{ }^{14}$. Esto explica que las muestras que recuperamos $\mathrm{y}$ procesamos para cultivo y frotis fueran negativas, $\mathrm{y}$ tal vez también influyeron los tratamientos antimicrobianos que habían recibido previamente las pacientes, mientras que las muestras evaluadas por RPC fueron positivas.

Por otro lado, cada vez es más común el empleo de técnicas moleculares para la identificación de $\mathrm{MNTs}^{15}$. En efecto, la presencia y disponibilidad de métodos de secuenciación de ADN, como la región ribosomal $16 \mathrm{~S}^{16}$, permite diferenciar especies con gran parentesco ${ }^{15}$. Mediante secuenciación se han diferenciado a especies altamente relacionadas, como $M$. abscessus y M. massiliense. De hecho, se cree que antes del advenimiento del diagnóstico molecular, muchas de las infecciones cuyo agente etiológico se estableció como $M$. abscessus, en realidad pudieron haber sido causadas por M. massiliense ${ }^{17}$.
Es difícil distinguir entre M. abscessus y M. massiliense con pruebas rutinarias de laboratorio clínico ${ }^{4}$. En cambio, M. massiliense es identificable por las secuencias de sus genes $r p o \beta$, $\operatorname{sod} A$, hsp65 y recA, por su secuencia espaciadora 16 23 del ARN ribosomal ${ }^{4,17}$, y por su secuencia genómica completa ${ }^{16}$. Además, aunque ambas especies poseen al gen de metiltransferasa ribosomal erm(41), en M. massiliense, el mismo se encuentra truncado y, por tanto, esta especie es sensible a macróli$\operatorname{dos}^{5}$. Adicionalmente, a diferencia de $M$. abscessus, $M$. massiliense es más sensible a claritromicina y no presenta un patrón uniforme de resistencia a doxiciclina ${ }^{4,17}$; a pesar de estos datos, no existe un tratamiento estándar para $M$. massiliense, por lo que su abordaje suele ser empírico ${ }^{17}$. En los casos que aquí comunicamos, la correcta identificación del agente permitió redirigir la terapia, evitando que las infecciones progresaran.

Las MNTs son bacterias ubicuas, y pueden colonizar la piel y los tractos respiratorio e intestinal de individuos sanos $^{15}$. Ya que tanto M. abscessus como M. massiliense son bacterias de baja virulencia, anteriormente se les asociaba con infecciones respiratorias o diseminadas en individuos inmunocomprometidos; no obstante, recientemente ha aumentado su incidencia en sujetos inmunocompetentes, en infecciones cutáneas y de tejidos blandos, asociados con trauma o inyecciones con sustancias contaminadas ${ }^{4,15}$. Nuestras pacientes entran en esta última categoría, pues todas eran inmunocompetentes y sin co-morbilidades.

Por otro lado, aunque la presentación clínica es variable $^{18}$, la presencia de M. massiliense se ha asociado con placas ulceradas o nódulos eritematosos y pápulas de larga evolución, asociados con dolor localizado y posibilidad de fistulización, que no responden a terapia antimicrobiana empírica para patógenos bacterianos comunes ${ }^{4}$.

En Colombia se reportaron 15 casos de pacientes infectados por MNTs secundario a mesoterapia, en donde la causa atribuible fue procaína contaminada ${ }^{19}$. En Francia se diagnosticaron 10 casos de infecciones subcutáneas graves por M. chelonae, uno por $M$. frederiksbergense, y otro por ambos, asociados al agua corriente de la habitación donde se realizó la técnica de mesoterapia ${ }^{20}$. En nuestro caso, una de las pacientes identificó un sobrenadante en el material que se aplicaron. No se tuvo éxito al intentar contactar a los fabricantes ni a los profesores del curso al que asistían las pacientes; además, es posible que no se cumpliera con una adecuada técnica aséptica, por lo que no fue posible definir si la infección la adquirieron por el material que emplearon o por una técnica deficiente de aplicación del PRP mediante mesoterapia.

Las infecciones por MNTs pueden ser confundidas con otras patologías, como inflamación granulomatosa supurativa y no supurativa. Infecciones como actinomicosis, coccidioidomicosis, nocardiosis y candidiasis, se asocian a granulomas supurativos y se identifican con 
tinciones como ácido peryódico de Schiff, metenamina de plata, Gomori-Grocott, mucicarmina, o Fontana-Masson, según sea el caso ${ }^{21}$. El pioderma gangrenoso, una dermatosis neutrofílica recurrente caracterizada por úlceras profundas con borde azul-violeta bien definido rodeado por piel indurada y eritematosa, acompañado por fiebre, malestar general, artralgias y mialgias, se asocia con condiciones sistémicas subyacentes como la enfermedad inflamatoria intestinal o neoplasias hematológicas ${ }^{19,22}$. El pioderma gangrenoso puede distinguirse de una infección cutánea por MNTs, por la identificación de anticuerpos anti-neutrófilos, mejoría con el tratamiento con inmunosupresores y cultivos sin crecimiento bacteriano ${ }^{22,23}$. Por otro lado, la sarcoidosis, condición inflamatoria idiopática y multiorgánica que afecta principalmente a mujeres afroamericanas, se caracteriza por granulomas no caseosos en diversos órganos, principalmente pulmones, con distribución broncovascular y linfática, y demuestran inclusiones inespecíficas como asteroides de Shaumann y cuerpos de Hamazaki-Wesenberg ${ }^{24}$. La sarcoidosis cicatricial, manifestación rara que se presenta en 2,9-29\% de los casos de sarcoidosis cutánea, se manifiesta como un edema con aspecto rojo purpúreo, y sucede en sitios de cicatrices antiguas tras daño local en la piel. Al igual que con la sarcoidosis generalizada, los granulomas no son caseosos y el tratamiento con corticoesteroides suprime la formación de granulomas y mejoran los síntomas ${ }^{25}$.

\section{Conclusiones}

Se presentan seis casos de pacientes con infección por M. massiliense tras la realización de mesoterapia. Los pacientes tratados con procedimientos estéticos como mesoterapia y aplicación de materiales de relleno, frecuentemente se atienden en escenarios sin adecuado control sanitario, y esto los pone en riesgo elevado para sufrir complicaciones. Por tal motivo, es necesario educar a los pacientes sobre la realización de prácticas cosmetológicas en sitios seguros y bajo correcta supervisión. Además, es obligatorio considerar múltiples posibilidades diagnósticas al momento de la consulta clínica en este tipo de pacientes. En los casos que reportamos, debido al antecedente de los tratamientos a los que fueron sometidas las pacientes, a la falta de respuesta a antibacterianos habituales, y a la heterogeneidad de las manifestaciones clínicas, se buscó intencionalmente una reacción granulomatosa asociada a cuerpo extraño, y se pudo identificar por RPC y secuenciación del producto amplificado a $M$. massiliense. Gracias a esto, se pudo dirigir la terapia hacia el agente etiológico con buenos resultados en la mayoría de las pacientes.

\section{Resumen}

Comunicamos seis casos de mujeres quienes, tras la aplicación mediante mesoterapia con plasma rico en plaquetas, así como de un material de relleno intradérmico de origen desconocido, desarrollaron una infección en los sitios de inyección asociada a Mycobacterium massiliense, así como granulomas con reacción a cuerpo extraño. Aunque los cultivos fueron negativos, se logró la identificación del microorganismo por extracción de ADN de tejidos blandos obtenido por biopsia y posterior secuenciación del producto obtenido. Debido a la gran similitud en los cultivos de $M$. massiliense con la especie relacionada Mycobacterium abscessus, y a que tienen diferente respuesta terapéutica, las técnicas moleculares de diagnóstico son una opción real a considerar para administrar en forma precoz el tratamiento específico contra el patógeno y evitar la progresión de la infección.

\section{Referencias bibliográficas}

1.- Herreros F O, Moraes A M, Velho P E. Mesotherapy: a bibliographical review. An Bras Dermatol 2011; 86: 96-101. doi:10.1590/s036505962011000100013.

2.- Atiyeh B S, Ibrahim A E, Dibo S A. Cosmetic mesotherapy: between scientific evidence, science fiction, and lucrative business. Aesthetic Plast Surg 2008; 32: 842-9. doi:10.1007/ s00266-008-9195-x.

3.- Sorlozano A, Soria I, Roman J, Huertas P, Soto M J, Piedrola G, et al. Comparative evaluation of three culture methods for the isolation of mycobacteria from clinical samples. J Microbiol Biotechnol 2009; 19: 1259-64.

4.- Cho A Y, Kim Y S, Kook Y H, Kim S O, Back $\mathrm{S} \mathrm{J}$, Seo Y J, et al. Identification of cutaneous
Mycobacterium massiliense infections associated with repeated surgical procedures. Ann Dermatol 2010; 22: 114-8. doi:10.5021/ ad.2010.22.1.114.

5.- Jeong S H, Kim S Y, Huh H J, Ki C S, Lee N Y, Kang C I, et al. Mycobacteriological characteristics and treatment outcomes in extrapulmonary Mycobacterium abscessus complex infections. Int J Infect Dis 2017; 60: 49-56. doi:10.1016/j.ijid.2017.05.007.

6.- Hachach-Haram N, Gregori M, Kirkpatrick N, Young R, Collier J. Complications of facial fillers: resource implications for NHS hospitals. BMJ Case Rep 2013. doi:10.1136/bcr-2012007141.

7.- Sturm L P, Cooter R D, Mutimer K L, Graham J C, Maddern G J. A systematic review of dermal fillers for age-related lines and wrinkles.
ANZ J Surg 2011; 81: 9-17. doi:10.1111/j.14452197.2010.05351.x.

8.- Wong S S, Wong S C, Yuen K Y. Infections associated with body modification. J Formos Med Assoc 2012; 111: 667-81. doi:10.1016/j. jfma.2012.10.016.

9.- Middleton K K, Barro V, Muller B, Terada S, Fu F H. Evaluation of the effects of platelet-rich plasma (PRP) therapy involved in the healing of sports-related soft tissue injuries. Iowa Orthop J 2012; 32: 150-63.

10.- Raeissadat S A, Sedighipour L, Rayegani S M, Bahrami M H, Bayat M, Rahimi R. Effect of Platelet-Rich Plasma (PRP) versus autologous whole blood on pain and function improvement in tennis elbow: a randomized clinical trial. Pain Res Treat 2014; 2014: 191525. doi:10.1155/2014/191525. 
11.- Davis M D, Wright T I, Shehan J M. A complication of mesotherapy: noninfectious granulomatous panniculitis. Arch Dermatol 2008; 144: 808-9. doi:10.1001/ archderm.144.6.808.

12.- Wagner D, Young L S. Nontuberculous mycobacterial infections: a clinical review. Infection 2004; 32: 257-70.

13.- Lamb R C, Dawn G. Cutaneous nontuberculous mycobacterial infections. Int $\mathrm{J}$ Dermatol 2014; 53: 1197-204. doi:10.1111/ ijd.12528.

14.- Fukunaga H, Murakami T, Gondo T, Sugi K, Ishihara T. Sensitivity of acid-fast staining for Mycobacterium tuberculosis in formalin-fixed tissue. Am J Respir Crit Care Med 2002; 166 : 994-7. doi: $10.1164 / \mathrm{rccm} .2111028$.

15.- Forbes B A, Hall G S, Miller M B, Novak S M, Rowlinson M C, Salfinger M, et al. Practice Guidelines for Clinical Microbiology Laboratories: Mycobacteria. Clin Microbiol Rev 2018; 31. doi:10.1128/ CMR.00038-17.

16.- Griffith D E, Brown-Elliott B A, Benwill J L, Wallace R J Jr. Mycobacterium abscessus.
"Pleased to meet you, hope you guess my name...”. Ann Am Thorac Soc 2015; 12: 436-9. doi:10.1513/AnnalsATS.201501-015OI .

17.- Jung M Y, Lee J H, Kim C R, Kim H J, Koh W J, Ki C S, et al. Cutaneous Mycobacterium massiliense infection of the sole of the feet. Ann Dermatol 2014; 26: 92-5. doi:10.5021/ ad.2014.26.1.92.

18.- Peters F, Batinica M, Plum G, Eming S A, Fabri $M$. Bug or no bug: challenges in diagnosing cutaneous mycobacterial infections. J Dtsch Dermatol Ges 2016; 14: 1227-35. doi:10.1111/ ddg. 13001.

19.- Sañudo A, Vallejo F, Sierra M, Hoyos J G, Yepes S, Wolff J C, et al. Nontuberculous mycobacteria infection after mesotherapy: preliminary report of 15 cases. Int J Dermatol 2007; 46: 649-53. doi: $10.1111 / j .1365$ 4632.2007.02976.x.

20.- Carbonne A, Brossier F, Arnaud I, Bougmiza I, Caumes E, Meningaud J P. Outbreak of nontuberculous mycobacterial subcutaneous infections related to multiple mesotherapy injections. J Clin Microbiol 2009; 47: 1961-64. doi:10.1128/JCM.00196-09.
21.- Fowler J, Mahlen S D. Localized cutaneous infections in immunocompetent individuals due to rapidly growing mycobacteria. Arch Pathol Lab Med 2014; 138: 1106-9. doi:10.5858/ arpa.2012-0203-RS.

22.- Brooklyn T, Dunnill G, Probert C. Diagnosis and treatment of pyoderma gangrenosum. BMJ 2006; 333: 181-4. doi:10.1136/ bmj.333.7560.181.

23.- Soncini J A, Grassi Salles A, Frizzo Neto J A, Gemperli R. Successful treatment of pyoderma gangrenosum after augmentation mastopexy using vacuum therapy. Plast Reconstr Surg Glob Open 2016; 4: e1072. doi:10.1097/ GOX.0000000000001072.

24.- Shah K K, Pritt B S, Alexander M P. Histopathologic review of granulomatous inflammation. J Clin Tuberc Other Mycobact Dis 2017; 7: 1-12 https://doi.org/10.1016/j. jctube.2017.02.001.

25.- Henrichs M P, Streitburger A, Gosheger G, Surke C, Dierkes C, Hardes J. Scar sarcoidosis on a finger mimicking a rapidly growing soft tissue tumour: a case report. BMC Res Notes 2012; 5: 545. doi:10.1186/1756-0500-5-545. 